\title{
Intermittency in presentation increases identification of masked figures ${ }^{1}$
}

\author{
KENNETH R. ALEXANDER ${ }^{2,3}$ \\ UNIVERSITY OF WASHINGTON
}

\begin{abstract}
Since low luminance nonsense figures projected onto visual noise tend to fade after onset, three groups of Os were used to determine if intermittency would aid pattern identification under such conditions. For the control group, patterns were superimposed on the noise for $5 \mathrm{sec}$. For the two experimental groups, an episcotister blade interrupted the patterns at rates of 2 and $8 \mathrm{~Hz}$ during the 5 -sec presentation. Intermittency significantly increased the number of patterns correctly identified, but there was no difference between the two rates.
\end{abstract}

Pattern identification is dependent on a number of parameters. The present study was designed to investigate the effects of intermittency on recognition when patterns are masked by visual noise. The stimuli and procedures were adapted from a study of the configurations of background, outline, and figure that result in maximum recognition under extremely poor viewing conditions (in preparation) The reduction in identifiability was accomplished by decreasing the luminance of the test stimuli and then superimposing them on a nonvarying pattern of visual noise, which was an out-of-focus mottled black and white surface. In the process of minimizing the probability of recognition, it was discovered that if a low-luminance target stimulus, of approximately 0.01 to $0.4 \mathrm{~mL}$, is projected onto the visual noise, of luminance $2.5 \mathrm{~mL}$, in a darkened room, the target stimulus is visible for a period of about $1 \mathrm{sec}$ following its onset, after which time it fades or disappears completely. For the stimulus to be visible, the $\mathrm{S}$ must observe it as it is first superimposed on the visual noise or as it leaves the noise at the offset of the stimulus. In the cases in which the figure disappears, once the figure is superimposed on the noise, no amount of scanning will facilitate perception of the stimulus-it is as if there were no figure in the noise.

Since the target stimuli fade or disappear after about $1 \mathrm{sec}$, it was hypothesized that intermittent presentation of the stimuli would facilitate identification. Furthermore, since faster rates of intermittency approach the CFF and phenomenally constant presentation, it would seem that a slower rate of interruption should be better for optimal identification than would a fast rate. Two rates of intermittency were arbitrarily chosen-one very slow and one nearer the CFF-to determine the effects of rate of flicker.

\section{Subjects \\ The Ss were 30 male and female} students, assigned randomly to the experimental conditions and run in groups of one to four.

\section{Apparatus}

The apparatus, as well as part of the procedure, was adapted from a study determining which combinations of background, outline, and figure luminances are most readily identifiable when figures are partly obscured by fixed visual noise. The target stimuli were $35-\mathrm{mm}$ slides of four randomly generated nonsense figures, identified by the letters $C, F, H$, and $R$. Each figure had a combination of one of four levels of background luminance, one of the same four levels of outline and figure luminances, and one of two widths of outline. The four levels of projected luminance were black $(0.01 \mathrm{~mL})$, dark gray $(0.05 \mathrm{~mL})$, light gray $(0.29 \mathrm{~mL})$, and white $(0.34 \mathrm{~mL})$. Excluding such combinations as white background, white outline, and white figure, in which there obviously are no identifiable shapes, this yields $\mathbf{4 8 0}$ different stimuli.

The projected stimuli, measured from one edge of the background to the other, subtended a visual angle of approximately $17 \mathrm{deg} 30 \mathrm{~min}$ when viewed from a distance of $4 \mathrm{~m}$. The slides of the stimuli were in random order, such that each figure occurred twice in a series of eight. Target figures were presented by a Kodak Carousel projector that had two partially rotated pieces of Bausch and Lomb polarizing film fitted over the lens, reducing the luminance levels to those given above. Projection distance was $5 \mathrm{~m}$. An episcotister blade, driven by one of two synchronous motors with speeds of 60 and $240 \mathrm{rpm}$, interrupted the projector beam near the lens to provide intermittency.

Visual noise consisted of a slide of irregular black shapes on a white field, projected by a second Kodak Carousel projector. The target stimuli were projected onto the visual noise, which was of an average luminance of $2.45 \mathrm{~mL}$. Since portions of the target stimuli cutting across the black shapes produced cues to the identity of the figures, through differential brightness, the noise was put slightly out of focus to eliminate such cues.

\section{Procedure}

The Ss were seated in a row in a darkened room at a viewing distance of $4 \mathrm{~m}$ from a white cardboard screen. In the training sequence, Ss were twice shown the last 32 slides in the series of 480 at the rate of $5 \mathrm{sec}$ per slide; the first time, Ss were told the correct identification of each figure, and the second time, they recorded their responses, with feedback from $E$ after each slide. The training series was presented without the visual noise. The Ss were then instructed to identify correctly as many of the stimuli in the experimental series as possible by recording the letter of the figure on an answer sheet arranged in blocks of eight responses. Ss were instructed to guess if they were uncertain of the correct response. Ilumination of the answer sheet was provided by ambient light from the two projectors. Slides were presented at a rate of $5 \mathrm{sec}$ per slide, with a pause after each set of 64 while slide trays were changed. At the beginning of each set of eight slides, the word "block" was pronounced, so that Ss could keep track of where they should be on the answer sheet.

The control group (GC) viewed the target stimuli superimposed on the visual noise without intermittency of the figures. For one experimental group (G120), each stimulus was presented with intermittency of 120 equal-interval on-off periods per minute. For the third group (G480), the stimuli were presented with intermittency of 480 on-off periods per minute.

Following the series of 480 target stimuli, the first 16 slides in the last tray of 32 were presented again without intermittency and without visual noise as a test series, to determine if Ss' labels for the figures corresponded to those designated by $E$. Data sheets of Ss who misidentified more than two of the test stimuli were discarded, as were data sheets of Ss who had consistently lost their place. A chi-square test of the number of data sheets rejected from each group was not significant $(p>.20)$.

\section{RESULTS}

The $F_{\max }$ test indicated that there was homogeneity of variance $(F=3.06$, 
$p>.05)$, so the total errors for each group were analyzed by Scheffé's test for unplanned comparisons. The differences between GC and G120 (F=16.64, $p<.01)$ and between $G C$ and $G 480$ $(F=26.34, p<.01)$ are significant. The difference between $\mathrm{G} 120$ and G480 $(F=1.11, p=n . s$.$) is not significant.$

\section{DISCUSSION}

As the results indicate, intermittency of the target stimuli at either rate significantly increased the number of figures correctly identified. The faster rate did not produce more errors, as predicted; in fact, there is a tendency, though not statistically significant, for the number of correct identifications to be larger for the faster rate.

One factor working against a difference between the control group and the two experimental groups was the inclusion of cues as to when the target stimuli would appear and disappear. At these times, the projector would click and an alert $S$ could have sufficient time to attend to the onset or offset of the figures. Also, since presentation time was the same for each figure, $S$ could have used time cues. The essential difference between the control and experimental groups was that $S$ s in the experimental groups saw the onset and offset of the figures many times during one stimulus presentation, instead of just once as in the control group. An alert $S$ in the control group could have scored the same as Ss in the other groups if he had attended to all the onsets and offsets of the figures. That this may have occurred is indicated by the fact that there is some tendency for the control group to overlap the other two. The onset-offset cues were not removed, however, since one of the purposes of the study was to see if intermittency would significantly improve recognition of figures under adverse conditions, and to determine if it would be profitable to provide intermittency in practical pattern-recognition tasks involving such conditions. The large differences suggest it would be helpful.

An explanation for the fading or disappearance of the target stimuli does not seem to be immediately available, but an examination of some of the parameters involved in the phenomenon would be useful for any attempt at an explanation. One of the major factors in the fading is target-figure luminance. As the luminance of the entire stimulus increases, there is a decrease in the amount of fading, until the figure is seen at all times with high luminance levels. With very low luminances, the stimuli are not visible at all. The luminance levels chosen for this experiment seemed to optimize the fading.

Focusing the visual noise also has an effect. With the noise completely in focus, there is some fading, but the cues arising from the figures covering some parts of the irregular black patterns allow the target stimulus to be seen fairly well. As the noise is moved more and more out of focus, there is an increase in the amount of fading and in difficulty in detecting the figures, until the noise is so far out of focus that the stimuli may again be seen fairly clearly.

Another variable affecting the disappearance is whether the stimuli or noise are moved during presentation. If either is moved, the figure is easily seen. The phenomenon seems to depend, then, on the projection of stationary stimuli onto fixed, out-of-focus visual noise. Complete figure disappearance occurs primarily with stimuli having light or white outlines and dark or black figures and background. The other combinations fade considerably, and transitions between the three stimulus regions of background, outline, and figure are generally obscured.

A possible reason for the difficulty in detecting the constantly presented stimuli is failure of the normal accommodation process due to the out-of-focus noise. This hypothesis seems unlikely, however, since there were abundant cues to the distance of both target stimuli and noise arising from ambient light reflected from the screen and room surfaces. The greater efficacy of the out-of-focus noise in obscuring the figures may indicate, instead, that contour processing is impaired if a contour of low luminance is superimposed on a background that itself has only luminance gradients and no abrupt luminance transitions. The phenomenon and its explanation bear further investigation.

\section{NOTES}

1. This report is based on a Master's thesis, University of Washington, 1968.

2. The author wishes to thank Dr. Sidney Culbert for his many helpful suggestions.

3. Address: Department of Psychology, University of Washington, Seattle, Washington 98105.

(Accepted for publication July 11, 1969.) 